\title{
FREQUÊNCIAS DO FORNECIMENTO DO ALIMENTO NO DESEMPENHO DE VACAS E NOVILHAS EM CONFINAMENTO
}

\author{
FREQUENCY OF THE SUPPLY OF FOOD ON THE PERFORMANCE OF COWS \\ AND HEIFERS IN FEEDLOT
}

\author{
Pazdiora, R.D. ${ }^{1 *}$; Pacheco, R.F. ${ }^{4}$; Brondani, I.B. ${ }^{2}$; Alves Filho, D.C. ${ }^{2}$; Menezes, L.F.G. ${ }^{3}$; \\ Callegaro, A.M. ${ }^{4}$; Pizzuti, L.A.D. ${ }^{4}$; Weise, M.S. ${ }^{4}$; Mayer, A. ${ }^{4}$ e Borchate, D. ${ }^{4}$
}

\begin{abstract}
${ }^{1}$ Departamento de Medicina Veterinária. Universidade Federal de Rondônia. UNIR. Campus Rolim de Moura. Brasil. *pazdiora@yahoo.com.br

${ }^{2}$ Departamento de Zootecnia. Universidade Federal de Santa Maria. UFSM. Santa Maria. Brasil.

${ }^{3}$ Departamento de Zootecnia. Universidade Tecnológica Federal do Paraná. UTFPR. Campus Dois Vizinhos. Brasil.

${ }^{4}$ Universidade Federal de Santa Maria. UFSM. Santa Maria. Brasil.
\end{abstract}

\section{PALAVRAS ChaVE ADICIONAIS}

Consumo. Conversão alimentar. Ganho de peso.

\section{RESUMO}

Objetivou-se avaliar a frequência do fornecimento do volumoso (V) e concentrado (C) no desempenho de vacas e novilhas em confinamento. Os tratamentos foram: $2 \mathrm{~V} / \mathrm{C}=\mathrm{V}$ e $\mathrm{C} 2$ vezes ao dia; $1 \mathrm{~V} / \mathrm{C}=\mathrm{V}$ e $\mathrm{C} 1 \mathrm{vez}$ ao dia; $1 \mathrm{~V} /$ $2 \mathrm{C}=\mathrm{V} 1$ vez e $\mathrm{C} 2$ vezes ao dia; $1 \mathrm{~V} / 3 \mathrm{C}=\mathrm{V} 1 \mathrm{vez}$ e $C 3$ vezes ao dia para vacas ou novilhas. $O$ delineamento experimental utilizado foi o inteiramente casualizado em arranjo fatorial $4 \times 2$, com 4 repetições. Não houve interação significativa entre frequência de fornecimento e categorias animal. O consumo de matéria seca (CMS) foi superior para os animais alimentados $1 \mathrm{~V} / 2 \mathrm{C}$ e 1 $\mathrm{V} / 3 \mathrm{C}$ ao dia em relação ao fornecimento de $2 \mathrm{~V} / \mathrm{C}$ e $1 \mathrm{~V} / \mathrm{C}$, apresentando valores de 11,$7 ; 11,4 ; 10,6$ e $10,7 \mathrm{~kg}$, respectivamente. O ganho de peso diário não foi influenciado pelas frequências de fornecimentos. O CMS foi superior para as vacas $(12,6 \mathrm{~kg})$ em relação às novilhas $(9,57 \mathrm{~kg})$, não havendo diferença para o ganho de peso. $\mathrm{O}$ aumento nas frequências do fornecimento do concentrado para 2 ou 3 vezes proporcionou maior consumo, não influenciando o ganho de peso.

\section{SUMMARY}

The effect of frequency of supply of forage

\section{AdDitional KeYWORDS}

Daily weight gain. Food conversion. Intake.

(V) and concentrate (C) on the performance of cows and heifers in feedlot was studied. The treatments were the following frequencies in the supply: $2 \mathrm{~V} / \mathrm{C}=\mathrm{V}$ and $\mathrm{C}$ twice daily; $1 \mathrm{~V} / \mathrm{C}=\mathrm{V}$ and $\mathrm{C}$ once daily; $1 \mathrm{~V} / 2 \mathrm{C}=$ once $\mathrm{V}$ and $\mathrm{C}$ twice daily, 1 $V / 3 C=$ once $V$ and $C$ three times a day, for the cows or heifers. The experimental design was completely randomized in factorial arrangement 4 $x 2$, with four replications. There was no significant interaction between frequency of supplying and category animal. The dry matter intake (DMI) was superior for the animals fed $1 \mathrm{~V} / 2 \mathrm{C}$ or $1 \mathrm{~V} / 3 \mathrm{C}$ a day in relation with the supply of $2 \mathrm{~V} / \mathrm{C}$ and $1 \mathrm{~V} / \mathrm{C}$, presenting values of $11.7 ; 11.4 ; 10.6$ and $10.7 \mathrm{~kg}$, respectively. The average daily gain (ADG) was not influenced by the frequency of supplying. The DMI was higher in cows $(12.6 \mathrm{~kg})$ than heifers $(9.57 \mathrm{~kg})$, having no difference in the weight gain. Increasing frequency of feeding the concentrate to two or three times a day, provided greater consumption, not influencing the weight gain of cows and heifers confined.

\section{INTRODUÇÃO}

O desempenho dos bovinos é decorrente, principalmente, do reflexo no manejo nutricional. Albright (1993) cita como 
fatores de alteração na quantidade de alimento ingeridos a localização da água, a acessibilidade da dieta, a competição entre os animais por espaço e alimento, além dos horários e das frequências de distribuição da dieta. Além disso, o animal pode regular seu consumo de matéria seca (CMS) por meio do ajuste do número e tamanho da refeição (Dado e Allen, 1994; Grant e Albright, 1995).

No entanto, dados encontrados na literatura são contraditórios com relação à frequência do fornecimento da dieta. No estudo de Gibson (1981), foi verificada vantagem para o ganho de peso (GMD) para o maior número de fornecimento do alimento aos bovinos. Porém, em outros estudos não encontraram diferença no CMS e GMD quando a alimentação foi fornecida em diferentes momentos ao longo do dia (Ruiz e Mowat, 1987; Goonewardene et al., 1995; Ferreira, 2006). Nestes trabalhos, as dietas foram distribuídas em partes iguais conforme o número de fornecimentos e não foi verificado aumento no CMS. Como o GMD apresenta elevada correlação com a ingestão de matéria seca (MS) (Reis et al., 2006), mais pesquisas devem ser realizadas no intuito de estimular maior consumo pelos animais e promover maior ganho de peso. Neste sentido, o fornecimento do concentrado fracionado ao longo do dia pode ser uma alternativa para a obtenção de maior consumo e exercer efeito positivo sobre a concentração de metabólitos ruminais, tendendo a reduzir os picos de ingestão e atividade fermentativa.

Além do manejo alimentar, a escolha da categoria animal também é importante. Apesar da baixa eficiência alimentar observada, a terminação de vacas para comercialização na entressafra tem mostrado boa rentabilidade principalmente pelo aumento do preço pago na primavera em relação ao verificado no outono (ANUALPEC, 2010). Por outro lado, a terminação de novilhas em confinamento pode ser uma opção mais viável em relação às vacas, já que a eficiência alimentar aumenta à medida que a idade dos animais decresce (Restle et al., 1998).

Neste sentido, objetivou-se avaliar a frequência do fornecimento do volumoso e concentrado no desempenho produtivo de vacas e novilhas.

\section{MATERIAL E MÉTODOS}

O experimento foi conduzido no Laboratório de Bovinocultura de Corte do Departamento de Zootecnia da Universidade Federal de Santa Maria (UFSM), situado na Depressão Central do Rio Grande do Sul.

Foram utilizadas 16 novilhas com idade média de 20 meses e peso médio de $338 \mathrm{~kg}$ e 16 vacas com idade média de 66 meses e 432 kg de peso médio ao início do período de avaliação, sendo os animais cruza Charolês Nelore.

Os animais foram confinados em baias de $20 \mathrm{~m}^{2}$ parcialmente cobertos, com piso de alvenaria, com bebedouro regulado por torneira-bóia e disponibilidade de $2 \mathrm{~m}$ linear de cocho por animal. Todos os boxes receberam dois animais de mesma categoria.

Foram adotados quatro manejos alimentares representados pelas frequências do fornecimento do volumoso e concentrado: $2 \mathrm{~V} / \mathrm{C}=$ volumoso e concentrado duas vezes ao dia fornecidos em parte iguais, às 8 e às 18 horas; $1 \mathrm{~V} / \mathrm{C}=$ volumoso e concentrado uma vez ao dia $(8 \mathrm{~h}) ; 1 \mathrm{~V} / 2 \mathrm{C}=$ volumoso uma vez ao dia ( $8 \mathrm{~h}$ ) e o concentrado duas vezes ao dia (8 e $18 \mathrm{~h}) ; 1 \mathrm{~V} / 3 \mathrm{C}=$ volumoso uma vez ao dia $(8 \mathrm{~h})$ e o concentrado três vezes ao dia $(8 ; 13$ e $18 \mathrm{~h})$, ofertados nas categorias vaca e novilha.

O confinamento teve a duração de 79 dias, sendo 16 dias de adaptação dos animais às instalações, manejo e alimentação e 63 dias de avaliações. A dieta fornecida aos animais foi composta de $60 \%$ de silagem de milho e $40 \%$ de concentrado com base na MS, misturados no cocho, com o objetivo inicial de atender à exigência de proteína bruta para GMD de 1,200 kg (NRC, 1996) estimando consumo de $2,5 \mathrm{~kg}$ de MS/100 kg 
de peso corporal (PC) (tabela I). A quantidade de alimento oferecida por dia foi regulada procurando-se manter sobras de $10 \%$ do ofertado. Para tanto, foram coletadas as sobras diariamente às $7 \mathrm{~h} 30 \mathrm{~min}$ a fim de medir o consumo do dia anterior e ajustar o fornecimento do dia subsequente.

Os animais foram pesados no início e ao final de cada período experimental (21 dias), após jejum de sólidos e líquidos de 14 h. No momento da pesagem foi avaliado o escore corporal, atribuindo escores de: $1=$ animal muito magro, $2=$ animal magro, $3=$ animal médio, 4= animal gordo e 5= animal muito gordo.

As amostras de silagem e sobras foram coletadas duas vezes por semana e o concentrado, semanalmente. As amostras foram secadas em estufa a $55{ }^{\circ} \mathrm{C}$ por $72 \mathrm{~h}$ para a

Tabela I. Composição da dieta utilizada no período experimental. (Diet composition used at experimental period).

\begin{tabular}{lcc}
\hline & Novilha & Vaca \\
\hline Componentes \% & & \\
Silagem de milho & 60,0 & 60,0 \\
Grão de milho & 10,0 & 6,0 \\
Farelo de trigo & 16,2 & 22,44 \\
Farelo de glúten de milho & 12,0 & 10,0 \\
Uréia & 0,48 & 0,32 \\
Calcário calcítico & 0,88 & 0,82 \\
Cloreto de sódio & 0,40 & 0,40 \\
Rumensin® & 0,016 & 0,012 \\
Sulfato de amônia & 0,03 & 0,02 \\
& & \\
Composição nutricional, \% & & \\
Matéria seca & 54,91 & 54,97 \\
Matéria orgânica & 90,09 & 90,07 \\
Proteína bruta & 12,89 & 12,10 \\
Extrato etéreo & 3,19 & 3,14 \\
Fibra em detergente neutro & 35,87 & 32,90 \\
Fibra em detergente ácido & 18,49 & 19,45 \\
Carboidratos não fibrosos & 38,14 & 41,93 \\
Nutrientes digestíveis totais & 71,53 & 71,66 \\
\hline
\end{tabular}

Valores expressos em $100 \%$ da MS. determinação da matéria parcialmente seca, após foram moídas em moinho tipo Willey, com peneira com crivos de $1 \mathrm{~mm}$ e armazenadas para posterior análise. A MS foi determinada em estufa a $105^{\circ} \mathrm{C}$ durante $16 \mathrm{~h}$. O conteúdo de cinzas foi determinado por combustão a $550{ }^{\circ} \mathrm{C}$ durante $2 \mathrm{~h}$ e o teor de matéria orgânica (MO) foi calculado com a diferença entre a MS e cinzas. O nitrogênio total foi determinado pelo método Kjeldahl (Método 984.13, AOAC, 1995), modificado por Kozloski et al. (2003). Os teores de lignina em detergente ácido (LDA) foram determinados de acordo com Robertson e Van Soest (1981). As determinações de fibra em detergente neutro (FDN) e fibra em detergente ácido (FDA) foram realizadas em saquinhos de poliéster (Komareck, 1993). Nitrogênio insolúvel em detergente ácido (NIDA) e nitrogênio insolúvel em detergente neutro (NIDN) foram analisados de acordo com Licitra et al. (1996). O extrato etéreo (EE) foi determinado em um sistema de refluxo com éter etílico, a $180^{\circ} \mathrm{C}$ durante 2 h (Soxtherm, Gerhardt, Alemanha). Os teores de carboidratos não-fibrosos (CNF) foram estimados segundo Mertens (1997), sendo CNF: $100-($ FDN\% + EE\% + PB\% + MM\%). O teor de nutrientes digestíveis totais (NDT) foi calculado segundo Weiss et al. (1992). A energia digestível (ED) foi calculada segundo NRC (1996), em que $1 \mathrm{~kg}$ de NDT=4,4 Mcal.

Para avaliar os coeficientes de digestibilidade foram conduzidos, paralelo ao experimento, dois ensaios de digestibilidade, em função das diferentes dietas utilizadas conforme a categoria animal. Os ensaios foram conduzidos com quatro bovinos fistulados no rúmen, em delineamento experimental tipo Quadrado Latino 4 x 4. Após um período pré-experimental de aproximadamente três semanas, com a finalidade de adaptar os animais ao manejo e às instalações, foi conduzido o experimento, em períodos de 15 dias, sendo os primeiros 10 dias destinados à adaptação dos animais às frequências do fornecimento e os cinco 
Tabela II. Consumos de matéria seca e energia digestível de volumoso (V) e concentrado (C) expresso em peso absoluto (CMS, CED), por $100 \mathrm{~kg}$ de peso corporal (CMSPC, CEDPC) $e$ por unidade de tamanho metabólico (CMSTM, CEDTM), consumo de fibra detergente neutro (CFDN) e conversão alimentar (CA). (Dry matter and digestible energy intake from roughage $(\mathrm{V})$ and concentrate (C) expressed by absolute weight, per $100 \mathrm{~kg}$ of body weight and per unit of metabolic size, neutral detergent fiber intake and food conversion).

\begin{tabular}{lcccccc}
\hline \multicolumn{7}{c}{ Frequências do fornecimento da dieta } \\
& 2 V/C & 1 V/C & 1 V/2C & 1 V/3C & Erro-padrão & $\mathrm{p}$ \\
\hline CMS, kg & 10,6 & 10,7 & 11,7 & 11,4 & 0,28 & 0,0665 \\
CMSPC, \% & 2,50 & 2,49 & 2,66 & 2,64 & 0,06 & 0,2012 \\
CMSTM, g & 113,6 & 113,1 & 121,9 & 119,9 & 2,93 & 0,1567 \\
CED, Mcal & 36,5 & 36,7 & 40,5 & 38,7 & 0,95 & 0,0742 \\
CEDPC, \% & 8,61 & 8,55 & 9,15 & 8,99 & 0,22 & 0,2341 \\
CEDTM, kcal & 0,39 & 0,38 & 0,41 & 0,40 & 0,01 & 0,1809 \\
CFDN, kg & 4,77 & 4,80 & 5,29 & 4,99 & 0,14 & 0,1190 \\
CA & 8,45 & 8,03 & 7,85 & 8,64 & 0,32 & 0,3439 \\
\hline
\end{tabular}

últimos dias à coleta de dados. Durante o período de adaptação os animais foram alimentados ad libitum, conforme as frequências do fornecimento e durante o período de coleta os animais receberam alimentação restrita, consumindo $90 \%$ do consumo voluntário. A digestibilidade aparente foi estimada através da coleta total das fezes dos animais, nos últimos cinco dias de cada período experimental, sendo retirada uma amostra, aproximadamente 10 $\%$ do total, diariamente. No último dia de cada período foram coletadas amostras de líquido ruminal de hora em hora durante 24 h, com exceção das 02, 03, 05, 06, 07 e 23 horas. Imediatamente após a coleta, as amostras foram filtradas e determinado o $\mathrm{pH}$ de cada amostra.

Não havendo interação para os coeficientes de digestibilidade entre a categoria animal e a frequência do fornecimento, os dados foram analisados somente para a frequência do fornecimento. Os dados da digestibilidade foram submetidos à análise de variância e as médias foram comparadas pelo teste $t$ de student (SAS, 2001), ao nível de $5 \%$ de significância.

Na avaliação do desempenho, o delineamento experimental utilizado foi o inteiramente casualizado em arranjo fatorial $4 \times 2$ (4 frequências de fornecimento e 2 categorias), utilizando-se 4 repetições. Os dados foram submetidos à análise de variância e as médias foram comparadas pelo teste $t$ de student (SAS, 2001), ao nível de 5 \% de significância.

\section{RESULTADOSEDISCUSSÃO}

Nas variáveis estudadas não houve interação entre as frequências do fornecimento da dieta e a categoria animal, sendo os dados analisados separadamente.

O CMS por animal (tabela II) foi superior para os animais alimentados com $1 \mathrm{~V} / 2 \mathrm{C}$ e $1 \mathrm{~V} / 3 \mathrm{C}$ ao dia em relação ao fornecimento de 2 V/C e 1 V/C (11,74; 11,37; 10,69 e 10,65 kg de MS, respectivamente), resultados explicados pelo estímulo ao consumo em função da presença de alimento de alta palatabilidade no cocho. No entanto, maior consumo não promoveu ( $\mathrm{p}>0,05)$ maior ganho de peso (tabela III), o que não era de se esperar, já que existe elevada correlação entre CMS e GMD (Reis et al., 2006). Em relação ao consumo de matéria seca por 100 kg de PC e por tamanho metabólico (TM), observou-se semelhança entre as fre- 
Tabela III. Pesos iniciais e finais, escores corporais iniciais e finais e ganhos médios diário em peso e escore corporal. (Initial and final weights, initial and final body conditions and average daily weight and body condition gains).

\begin{tabular}{|c|c|c|c|c|c|c|}
\hline & \multicolumn{4}{|c|}{ Frequências do fornecimento da dieta } & \multirow[b]{2}{*}{ Erro-padrão } & \multirow[b]{2}{*}{$p$} \\
\hline & $2 \mathrm{~V} / \mathrm{C}$ & $1 \mathrm{~V} / \mathrm{C}$ & $1 \mathrm{~V} / 2 \mathrm{C}$ & $1 \mathrm{~V} / 3 \mathrm{C}$ & & \\
\hline Peso inicial, kg & 381 & 383 & 391 & 385 & 11,0 & 0,9229 \\
\hline Peso final, kg & 460 & 469 & 485 & 467 & 11,8 & 0,5366 \\
\hline Escore corporal inicial, pontos* & 2,98 & 2,90 & 2,94 & 2,97 & 0,04 & 0,6013 \\
\hline Escore corporal final, pontos* & 3,57 & 3,52 & 3,59 & 3,62 & 0,06 & 0,7294 \\
\hline Ganho médio diário, kg & 1,265 & 1,360 & 1,486 & 1,307 & 0,08 & 0,2697 \\
\hline Ganho de escore corporal, pontos ${ }^{1}$ & 0,58 & 0,61 & 0,64 & 0,65 & 0,04 & 0,7617 \\
\hline
\end{tabular}

$\mathrm{V}=$ volumoso $\mathrm{C}=$ concentrado.

*1= animal muito magro; 2= animal magro; 3= animal médio; 4= animal gordo e 5= animal muito gordo.

quências de fornecimento.

O consumo de energia digestível (CED) e fibra detergente neutro (CFDN) apresentam a mesma tendência do CMS. Como os componentes da dieta foram iguais, os maiores CED e CFDN devem-se ao maior CMS da dieta. Ruiz e Mowat (1987), não encontraram efeito do número de alimentação (uma ou quatro vezes) sobre o consumo, quando a alimentação foi oferecida à vontade. Segundo os autores, o padrão de consumo permanece constante durante o dia, independente do número de fornecimento, quando a alimentação é oferecida à vontade. $\mathrm{O}$ aumento do número de alimentação pode ser benéfico no caso de alimentação restrita, quando os animais modificam o padrão de consumo, ingerindo grande quantidade logo após o fornecimento, como observado em bovinos submetidos ao pastejo restrito em pastagens cultivadas de inverno (Arboitte et al., 2006).

A conversão alimentar (tabela II) foi similar nas quatro frequências de alimentação. Vários autores (Kaufmann, 1976; French e Kennely, 1984; Robinson e Mcniven, 1994) consideraram que o aumento no número de refeições tem reflexo positivo no desempenho animal, por melhorar a fermentação ruminal, através da redução das flutuações dos ácidos graxos voláteis, $\mathrm{pH}$, amônia, elevando a digestão da fibra, porém isto não ocorreu no presente trabalho.

O desempenho não foi influenciado ( $p>0,05)$ pelas diferentes frequências de fornecimento da dieta (tabela III). O fornecimento do volumoso pela manhã e o fornecimento do concentrado uma, duas ou três vezes ao dia foram 7,5; 17,5 e 3,3 \% a mais, porém não significativos ( $p>0,05$ ), para o ganho de peso, em relação ao fornecimento do volumoso e do concentrado duas vezes ao dia. Fornecendo duas, três ou quatro vezes a alimentação diariamente, Ferreira (2006) verificou que não houve efeito do aumento do número de vezes em que foi fornecido o alimento sobre o GMD e estado corporal. O autor justifica o ganho similar entre as três frequências de alimentação em função do CMS diário ter sido similar entre os fornecimentos. Segundo Colemen e Wyatt (1984) e Sniffen e Robinson (1984), o aumento em GMD e eficiência ocorrem quando o aumento da frequência de alimentação promove elevação no consumo, o que também pode estar relacionado à melhor uniformidade nas concentrações de metabólicos ruminais.

A ausência do efeito da frequência de alimentação sobre o desempenho produtivo 
Tabela IV. Coeficientes de digestibilidade aparente da matéria seca (DAMS), matéria orgânica (DAMO), da fibra detergente neutro (DAFDN) e verdadeira da matéria orgânica (DVMO). (Apparent digestibility coefficients of dry matter, organic matter, neutral fiber detergent and real organic matter).

\begin{tabular}{lcccccc}
\hline \multicolumn{7}{c}{ Frequências do fornecimento da dieta } \\
& 2 V/C & 1 V/C & 1 V/2C & 1 V/3C & Erro-padrão & $\mathrm{p}$ \\
\hline CMS, kg & 10,6 & 10,7 & 11,7 & 11,4 & 0,28 & 0,0665 \\
CMSPC, \% & 2,50 & 2,49 & 2,66 & 2,64 & 0,06 & 0,2012 \\
CMSTM, g & 113,6 & 113,1 & 121,9 & 119,9 & 2,93 & 0,1567 \\
CED, Mcal & 36,5 & 36,7 & 40,5 & 38,7 & 0,95 & 0,0742 \\
CEDPC, \% & 8,61 & 8,55 & 9,15 & 8,99 & 0,22 & 0,2341 \\
CEDTM, kcal & 0,39 & 0,38 & 0,41 & 0,40 & 0,01 & 0,1809 \\
CFDN, kg & 4,77 & 4,80 & 5,29 & 4,99 & 0,14 & 0,1190 \\
CA & 8,45 & 8,03 & 7,85 & 8,64 & 0,32 & 0,3439 \\
DAMS & 64,8 & 67,7 & 66,9 & 65,5 & 1,35 & 0,4393 \\
DAMO & 64,9 & 68,1 & 67,6 & 66,1 & 1,33 & 0,3402 \\
DAFDN & 55,3 & 59,6 & 59,0 & 58,1 & 1,86 & 0,3959 \\
DVMO & 75,5 & 77,8 & 77,6 & 77,0 & 1,01 & 0,3886 \\
\hline
\end{tabular}

$\mathrm{V}=$ volumoso; $\mathrm{C}=$ concentrado.

também foi constatada por Goonewardene et al. (1995), que realizaram estudo comparando CMS, GMD e eficiência alimentar com bovinos recebendo uma, duas ou três alimentações ao dia. Aspecto importante sob ponto de vista prático, com relação à frequência de alimentação, é o seu custo, demonstrado por Restle et al. (2007) que ao estudarem a avaliação econômica da terminação em confinamento de novilhos, verificaram que dois fornecimentos diários, normalmente empregado no sul do Brasil, representou 9,6\% do custo operacional da terminação, quando excluído o valor dos animais. Para Ferreira (2006), aumentar o número de fornecimentos diários implica no aumento no custo de produção e somente seria justificado caso resultasse no incremento do desempenho animal. Segundo Pacheco et al. (2006), espera-se grande variação nos custos com o fornecimento da alimentação, pois a quantidade fornecida normalmente está relacionada à dimensão dos cochos, que determina o número de fornecimentos diários. Assim, em virtude da importância de sua participação nos custos de produção, é sugerido o planejamento prévio das instalações objetivando maximização técnica e econômica desta operação.

No presente estudo, os coeficientes de digestibilidade não foram influenciados ( $>>0,05)$ pelas frequências da alimentação (tabela IV). No entanto, estes coeficientes podem estar mascarados por uma restrição do consumo voluntário da dieta no período de coleta, o que não ocorreu durante a terminação dos animais e a similaridade no GMD pode estar relacionada à digestibilidade.

Maior GMD para animais alimentados com maior frequência diária de alimentação é atribuído por Ikhatua et al. (1987) aos maiores coeficientes de digestibilidade da MS, MO e fibra bruta. Em seu estudo, a digestibilidade dos nutrientes aumentou apenas quando se elevou o número de fornecimentos de uma para duas vezes ao dia, não havendo diferença quando o número de refeições passou de duas para três 
vezes ao dia. Tal diferença não foi observada no presente estudo.

$\mathrm{O}$ pH teve variação entre os fornecimentos, conforme a figura 1. Após a alimentação dos animais pela manhã (8 h), observa-se queda no valor do $\mathrm{pH}$ ruminal para todas as frequências de fornecimento, porém, para os animais alimentados duas vezes com o volumoso e concentrado verifica-se menor variação ao longo do dia. Posteriormente ao pico de digestão dos alimentos, observa-se elevação dos valores de $\mathrm{pH}$ até o fornecimento da alimentação às $18 \mathrm{~h}$. O fornecimento do concentrado as 13 h, não proporcionou maior queda do $\mathrm{pH}$ ruminal nos animais que receberam $1 \mathrm{vez}$ volumoso e 3 vezes o concentrado/dia.

Elevadas proporções de concentrado na dieta de ruminantes pode refletir em ingestão muito rápida de grande quantidade de carboidratos solúveis, o que poderia provocar distúrbios digestivos nos animais, pois o consumo do amido associado ao declínio no pH ruminal afetam as bactérias ruminais, resultando na redução da digestão da fibra e do consumo da dieta total (Caton e Dhuyvetter, 1997). Valores de $\mathrm{pH}$ inferiores a 6,2 inibem a taxa de digestão e aumentam o tempo de colonização para a degradação celular (Van Soest, 1994).

$\mathrm{Na}$ avaliação das categorias animais, os CMS e CED por animal e por tamanho metabólico foram superiores para as vacas (tabela V), pela maior capacidade de ingestão dessas, dado a grande diferença de PC em relação às novilhas. As vacas consumiram $32 \%$ mais MS que as novilhas. No presente estudo, o consumo foi semelhante ao encontrado por Ferreira (2006), que verificou consumo de $29 \%$ a mais de MS para as vacas em relação aos novilhos.

Em relação à porcentagem de PC, o consumo não diferiu entre as categorias. Restle et al. (2001) observaram que as novilhas consumiram menos $(\mathrm{p}<0,01)$ MS que as vacas $(11,61$ vs 13,17 kg/animal/dia, respecti-

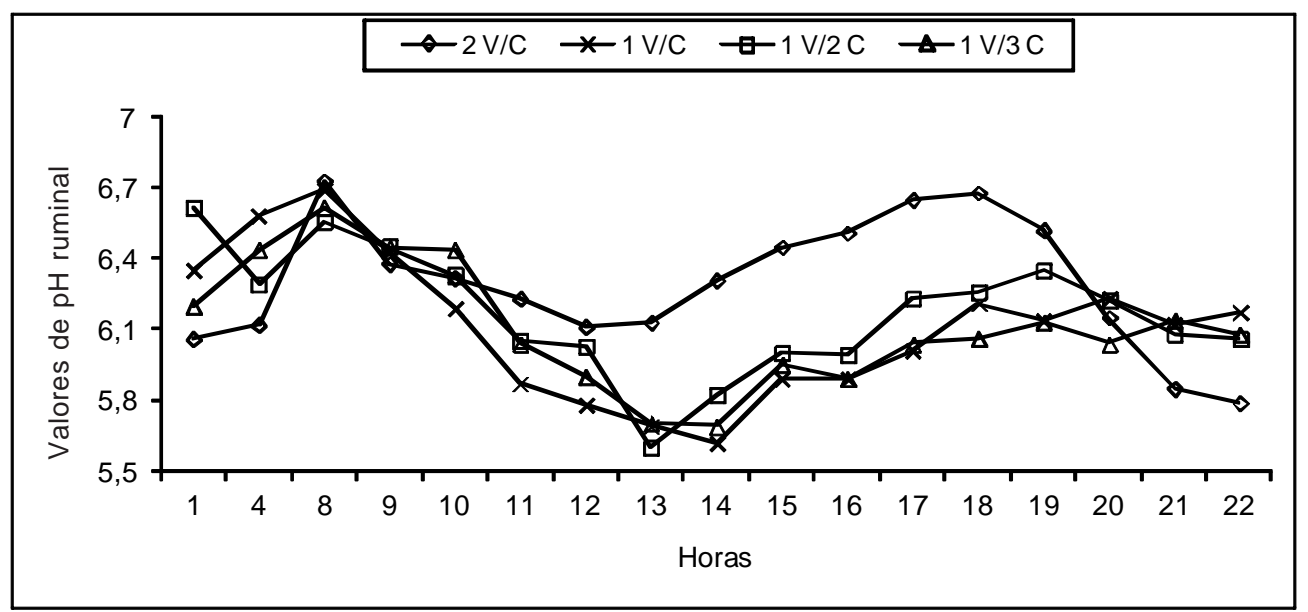

$2 \mathrm{~V} / \mathrm{C}=$ volumoso e concentrado duas vezes ao dia fornecidos em parte iguais, às 8 e às 18 horas. $1 \mathrm{~V} / \mathrm{C}=$ volumoso e concentrado uma vez ao dia $(8 \mathrm{~h})$.

$1 \mathrm{~V} / 2 \mathrm{C}=$ volumoso uma vez ao dia $(8 \mathrm{~h})$ e o concentrado duas vezes ao dia (8 e $18 \mathrm{~h}$ ).

$1 \mathrm{~V} / 3 \mathrm{C}=$ volumoso uma vez ao dia $(8 \mathrm{~h})$ e o concentrado três vezes ao dia ( 8 ; 13 e $18 \mathrm{~h}$ ).

Figura 1. Variação do pH ao longo do dia conforme a frequência do fornecimento da dieta. (Variation of the $\mathrm{pH}$ throughout the day according to the frequency of the supply of the diet). 
Tabela V. Consumos diário de matéria seca e energia digestível expressos por animal (CMS, CED), por $100 \mathrm{~kg}$ de peso corporal (CMSPC, CEDPC) e por unidade de tamanho metabólico (CMSTM, CEDTM), consumo de fibra detergente neutro (CFDN) e conversão alimentar (CA). (Dry matter and digestible energy daily intake expressed by animal, per $100 \mathrm{~kg}$ of body weight and per unit of metabolic size, neutral detergent fiber intake and food conversion).

\begin{tabular}{|c|c|c|c|c|}
\hline & Novilha & Vaca & ro-padrão & $\mathrm{p}$ \\
\hline CMS, kg & 9,57 & 12,65 & 0,19 & $<0,0001$ \\
\hline CMSPC, \% & 2,51 & 2,64 & 0,04 & 0,0722 \\
\hline CMSTM,g & 110,8 & 123,5 & 2,07 & 0,0026 \\
\hline CED, Mcal & 33,6 & 42,6 & 0,67 & $<0,0001$ \\
\hline CEDPC, $\%$ & 8,77 & 8,88 & 0,15 & 0,6122 \\
\hline CEDTM, kcal & 0,387 & 0,415 & 0,007 & 0,0235 \\
\hline CFDN, kg & 4,10 & 5,82 & 0,10 & $<0,0001$ \\
\hline CA & 7,23 & 9,26 & 0,22 & 0,0003 \\
\hline
\end{tabular}

vamente), porém, essa diferença desapareceu quando se avaliou o CMS por 100 $\mathrm{kg}$ de $\mathrm{PC}$ e por TM (p>0,05), mostrando que o consumo aumentou em função do peso dos animais.

As novilhas foram mais eficientes $(\mathrm{p}<0,05)$ em converter o alimento em $\mathrm{kg}$ de PC do que as vacas, pois para cada $\mathrm{kg}$ de ganho, consumiram 7,4 vs 9,4 kg de MS, respectivamente. A diferença de $27 \%$ na conversão alimentar deve-se ao fato das vacas, por serem mais pesadas e apresentarem maior TM, necessitarem de maior aporte de nutrientes para atender às exigências energéticas de mantença. Outro fator que implica na eficiência da conversão é a composição do ganho, já que o ganho de peso em animais jovens é devido, em parte, ao crescimento dos tecidos musculares, estriado e liso, dos órgãos vitais e do tecido ósseo, que exigem mais proteína para o seu crescimento; nos animais adultos o ganho consiste, basicamente, no depósito de gordura, com exigências energéticas maiores (Di Marco, 1994).

Ferreira (2006) observou que a eficiência em transformar MS e energia digestível (ED) em GMD foi afetada significativamente pela categoria animal. Este autor verificou que, apesar do maior GMD das vacas em relação aos novilhos (1,60 vs 1,45 kg, respectivamente), elas foram menos eficientes na transformação da MS e da energia em ganho de peso que os novilhos (9,30 vs 7,90 kg de MS/kg de ganho e 27,47 vs 23,42 kg de ED/ $\mathrm{kg}$ de ganho, respectivamente). Restle et al. (2001) verificaram que as novilhas foram mais eficientes $(p<0,01)$ que as vacas, necessitando de 6,40 kg de MS de alimento para produzir $1 \mathrm{~kg}$ de ganho de peso enquanto que as vacas necessitaram de 8,27 kg.

As vacas apresentaram maiores pesos

Tabela VI. Pesos iniciais e finais, escores iniciais e finais e ganhos médios diário em peso e escore de acordo com a categoria. (Initial and final weights, initial and final body conditions and average daily weight and body condition gains according to animal category).

\begin{tabular}{lcccc}
\hline & Novilha & Vaca & Erro-padrão & $\mathrm{p}$ \\
\hline Peso inicial, $\mathrm{kg}$ & 338 & 432 & 7,80 & $<0,0001$ \\
Peso final, $\mathrm{kg}^{\text {Escore corporal inicial, pontos }}{ }^{1}$ & 421 & 519 & 8,38 & $<0,0001$ \\
Escore corporal final, pontos $^{1}$ & 2,97 & 2,92 & 0,03 & 0,2932 \\
Ganho médio diário, $\mathrm{kg}^{\text {Ganho de escore corporal, pontos }}{ }^{1}$ & 3,50 & 3,65 & 0,04 & 0,0192 \\
& 1,330 & 1,378 & 0,05 & 0,5569 \\
\hline
\end{tabular}

${ }^{1} 1=$ animal muito magro; 2= animal magro; 3= animal médio; 4= animal gordo e 5= animal muito gordo. 
inicial e final (tabela VI). Isto já era esperado, em função das novilhas ainda estarem em fase de crescimento e não terem atingido o peso adulto.

O GMD foi semelhante para as novilhas e vacas $(1,330$ vs $1,378 \mathrm{~kg}$, respectivamente), mostrando a boa eficiência para ganho de peso do bovino jovem comparado àquele de maior idade. Restle et al.(2001), avaliando o desempenho de novilhas e vacas em confinamento, verificaram similaridade no GMD entre as categorias, sendo os valores observados 1,88 e $1,73 \mathrm{~kg}$, respectivamente, os ganhos observados por estes autores foram 0,41 e $0,25 \%$ superiores aos verificados no presente estudo. As vacas apresentaram maior estado corporal final e ganho de estado corporal. Isto ocorreu porque as novilhas apresentavam crescimento muscular e ósseo, acumulando mais tecido muscular e menos tecido adiposo.

\section{BIBLIOGRAFIA}

Albright, J.L. 1993. Nutrition, feeding and calves. Feeding behavior of dairy cattle. J Dairy Sci, 76: 485-498.

ANUALPEC. 2010. Anuário da pecuária brasileira. Agra FNP pesquisas Ltda. São Paulo. 380 pp.

Arboitte, M.Z.; Restle, J.; Brondani, I.L.; Menezes, L.F.G.; Missio, R. e Segabinazi, L.R. 2006. Pastejo contínuo ou temporário e suplementação energética em pastagem cultivada de inverno no desempenho de bezerros. Acta Sci Anim Sci, 28: 453-459.

AOAC. Association of Official Analytical Chemists. 1995. Official methods of analysis. $12^{\mathrm{a}}$ ed. Washington, D.C.

Caton, J.S. and Dhuyvetter, D.V. 1997.Influence of energy supplementation on grazing ruminants: requirements and responses. J Anim Sci, 75: 533-542.

Colemen, S.W. and Wyatt, R.D. 1984. Cottonseed meal or small grains forages as protein supplements fed at different intervals. J Anim Sci, 77: 132-144.

Dado, R.G. and Allen, M.S. 1994. Variation in and relationships among feeding, chewing, and drinking variables for lacting dairy cows. $J$
Segundo Owens (1993), a ordem de crescimento dos tecidos se dá, primeiramente, pelo tecido ósseo, seguido do muscular e posteriormente o tecido adiposo.

\section{CONCLUSÃO}

O aumento na frequência do fornecimento do concentrado para duas ou três vezes ao dia proporciona maior consumo de alimento. No entanto, não influencia o ganho de peso dos animais confinados em comparação ao fornecimento da alimentação uma vez ou o fornecimento do volumoso e o concentrado duas vezes ao dia, revelando-se prática não recomendada para fêmeas confinadas.

Vacas são menos eficientes na transformação do alimento em ganho de peso, apresentando consumo superior e ganho de peso similar em relação às novilhas.

Dairy Sci, 77: 132-144.

Di Marco, O.N. 1994. Crescimiento y respuesta animal. Associación Argentina de Producción Animal. Buenos Aires. 129 pp.

Ferreira, J.J. 2006. Desempenho e comportamento ingestivo de novilhos e vacas sob frequências de alimentação em confinamento. Dissertação de Mestrado. Universidade Federal de Santa Maria. Santa Maria, RS.

French, N. and Kennelly, J.J. 1984. The effect of frequency of feeding on rumen parameters and on blood insulin concentrations in dairy cows. J Anim Sci, 64: 1072-1075.

Gibson, J.O. 1981. The effects of feeding frequency on growth and efficiency of food utilization of ruminants. Anim Prod, 32: 275-283.

Goonewardene, L.A.; Zobell, D.R. and Engstrom, D.F. 1995. Feeding frequency and its effect on feedlot performance in steers. J Anim Sci, 75: 255-257.

Grant, R.J. and Albright, J.L. 1995. Feeding behavior and management factors during the transition period in dairy cattle. J Anim Sci, 73: 2791-2803.

Ikhatua, U.J.; Ehoche, O.W. and Umoh, J.E. 1987. 


\section{PAZDIORAETAL.}

The influence of feeding frequency on feed intake, nutrient utilization and nitrogen metabolism in growing zebu cattle. J Agric Sci, 108: 639-642.

Kaufmann, W. 1976. Influence of the composition of the ration and the feeding frequency on ph regulation in the rumen and on feed intake in ruminants. Livest Prod Sci, 3: 103-114.

Komarek, A.R. 1993. A fiber bag procedure for improved efficiency of fiber analyses. J Dairy Sci, 76: 250.

Kozloski, G.V.; Perottoni, J. and Rocha, J.B.T. 2003. Potencial nutricional assessment of dwarf elephant grass (Pennisetum purpureum Schum. 'Mott') by chemical composition, digestion and net portal flux of oxygen in cattle. Anim Feed Sci Technol, 104: 29-40.

Licitra, G.; Hernandez, T.M. and Van Soest, P.J. 1996. Standartization of procedures for nitrogen fractionation of ruminant feeds. Anim Feed Sci Technol, 57: 347-358.

NRC. National Research Council. 1996. Nutrient requeriment of beef cattle. $7^{\mathrm{a}}$ ed. National Academy Press. Washington. 242 pp.

Owens, F.N.; Dubeski, P. and Hanson, C.F. 1993. Factors that alter the growth and development of ruminants. J Anim Sci, 71: 3138-3150.

Pacheco, P.S.; Restle, J.; Vaz, F.N. ; Freitas, A.K.; Padua, J.T.; Neumann, M. e Arboitte, M.Z. 2006. Avaliação econômica da terminação em confinamento de novilhos jovens e superjovens de diferentes grupos genéticos. Rev Bras Zootecn, 35: 309-320.

Reis, R.A.; Nussio, L.G.; Coan, R.M.; Resende, F.D. e Signoretti, R. 2006. Adequação do uso de alimentos volumosos: custos de produção e desempenho comparativo. Coanconsultoria, SP. Disponível em <http://www.coanconsultoria. com.br/> (15/05/2008).
Restle, J.; Lupatini, G.C.; Roso, C. e Soares, A.B. 1998. Eficiência e desempenho de diferentes categorias de bovinos de corte em pastagem cultivada. Rev Bras Zootecn, 27: 397-404.

Restle, J.; Neumann, M.; Alves Filho, D.C.; Pascoal, L.L.; Rosa, J.R.P.; Menezes, L.F.G. e Pellegrini, L.G. 2001. Terminação em confinamento de vacas e novilhas sob dietas com ou sem monensina sódica. Rev Bras Zootecn, 30: 18011812.

Restle, J.; Pacheco, P.S.; Costa, E.C., Freitas, A.K.; Vaz, F.N.; Brondani, I.L. e Fernandes, J.J.R. 2007. Apreciação econômica da terminação em confinamento de novilhos Red Angus superjovens, abatidos com diferentes pesos. Rev Bras Zootecn, 34: 978-986.

Robertson, J.B. and Van Soest, P.J. 1981. The detergent system of analysis. In: James, W.P.T. and Theander, O. (Eds.). The analysis of dietary fibre in food. Marcel Dekker. New York. pp. 123158.

Robinson, P.H. and Mcniven, M.A. 1994. Influence of flame roasting and feeding frequency of barley on performance of dairy cows. J Dairy Sci, 77: 3631-3643.

Ruiz, A. and Mowat, D.N. 1987. Effect of feeding frequency on the utilization of high forage diets by cattle. J Anim Sci, 67: 1067-1074.

SAS, Institute Inc. 2001. SAS Language Reference. Version 6. SAS institute. Cary, NC. 1042 pp.

Sniffen, C.J. and Robinson, P.H. 1984. Nutritional strategy. J Anim Sci, 64: 529-542.

Van Soest, P.J. 1994. Nutritional ecology of the ruminant. $2^{\mathrm{a}}$ ed. Cornell University Press. Ithaca, NY. $476 \mathrm{pp}$.

Weiss, W.P.; Conrad, H.R. and Pierre, N.R. 1992. A theoretically-based model for predicting total digestible nutrient values of forages and concentrates. Anim Feed Sci Technol, 39: 95-110.

Archivos de zootecnia vol. 63, núm. 241, p. 12. 